\title{
Research Paper \\ The Effect of Selected Corrective Exercises With Physioball on the Posture of Female Computer Users With Upper Crossed Syndrome
}

\author{
Fariba Javazi ${ }^{1}\left(\mathbb{D}\right.$, *Parisa Sedaghati $^{1}\left(\mathbb{D}\right.$, Hasan Daneshmandi ${ }^{1}$
}

1. Department of Sport Injuries and Corrective Exercise, Faculty of Physical Education and Sport Sciences, University of Guilan, Rasht, Iran.

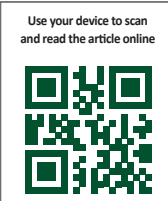

Cittation: Javazi F, Sedaghati P, Daneshmandi H. [The Effect of Selected Corrective Exercises With Physioball on the Posture of Female Computer Users With Upper Crossed Syndrome (Persian)]. Journal of Sport Biomechanics. 2019; 5(2):112-123. https://doi.org/10.32598/biomechanics.5.2.5

doi https://doi.org/10.32598/biomechanics.5.2.5

Key words:

Corrective exercises, Upper crossed syndrome, Physioball, Computer users

\section{ABSTRACT}

Objective Upper Crossed Syndrome (UCS) is a type of musculoskeletal system involvement that results in shortening of anterior muscles and weakening of posterior muscles. The aim of this study was to examine the effect of selected corrective exercises with physioball on the postural status of female computer users with UCS.

Methods This quasi-experimental study was conducted on 24 female students with UCS who were selected using a purposive sampling method. The subjects were randomly divided into two groups of exercise $(n=12)$ and control $(n=12)$. The exercise group performed exercises for 6 weeks. Photogrammetry method was used to measure the angle of the forward head and round shoulder. A flexible ruler was used to measure the angle of kyphosis, and tape measure was used to measure the chest expansion. These measurements were performed before and after intervention. The collected data were analyzed using paired $t$-test and ANOVA at the significance level of $P<0.05$.

Results There was a significant difference in mean values of kyphosis $(P=0.001)$, round shoulder $(P=0.001)$, forward head $(\mathrm{P}=0.002)$ and chest expansion $(\mathrm{P}=0.003)$ before and after exercise.

Conclusion Improvement in forward head, round shoulder and thoracic kyphosis angles and chest expansion showed the effectiveness of applied exercise program. Therefore, it is recommended to use this exercise program in computer users with UCS.

\section{Extended Abstract}

\section{Introduction}

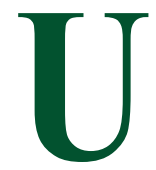

pper Crossed Syndrome (UCS) is a type of musculoskeletal system involvement that results in shortening of the anterior muscles and weakening of the posterior muscles. Certain postural abnormalities are seen in those with UCS which includes the forward head, rounded shoulders, and thoracic kyphosis. This disorder has been developed due to the change of lifestyle from active to inactive. In today's population, UCS is very common among all age groups. According to evidences, the prevalence of UCS is also high among the student community.

One of the tools that have forced people to work for hours without mobility is computer. Students are among those who spend many hours per day working in front of a computer, where the upper body posture especially the neck,

* Corresponding Author:

Parisa Sedaghati, PhD.

Address: Department of Sport Injuries and Corrective Exercise, Faculty of Physical Education and Sport Sciences, University of Guilan, Rasht, Iran. Tel: +98 (13) 33690161

E-mail: parisasedaghati@yahoo.com 
are misaligned which predisposes a person to upper limb abnormalities. In the spine region, hyperkyphosis means an increase in the kyphosis angle, which also affects the cervical and lordotic curvatures and causes the trunk to move forward more.

The abnormal tendency of the trunk forward in kyphosis reduces the supportive role and flexibility of the spine, and causes chest, lung, and heart problems. Therefore, it is necessary to pay attention to the inappropriate postural conditions of students as the national assets of the country. Exercise in different conditions and with different tools can have a great impact on correcting posture. One of these exercises is physioball exercise. Using physioball can increase the activity of the muscles that produce movement, and this increase is in the muscles of different parts, including the shoulder girdle [21], lower limbs and trunk muscles. More muscle involvement during exercise is one of the most important goals of each type of exercise. The aim of the present study was to investigate the effect of selected corrective exercises with physioball on the posture of computer users with UCS.

\section{Participants and Methods}

This is a quasi-experimental study with pretest/posttest design. Twenty-four female computer users with head forward, round shoulder, and thoracic kyphosis were selected using purposive sampling method and randomly divided into two control $(n=12)$ and exercise groups $(n=12)$. photogrammetry method was used to measure the angle of forward head and round shoulder at the same time, and a flexible ruler was used to measure the degree of thoracic kyphosis. To measure chest expansion, the chest circumference was first measured at the end of the exhalation with a tape. The subject was then asked to take a deep breath. The chest circumference was measured again and the difference between the two measurement values was recorded. The change in forward head, round shoulder, thoracic kyphosis, and chest expansion were measured before and after exercise. ANOVA and paired t-test were used to analyze the data.

\section{Results}

The results of the present study showed a significant difference in thoracic kyphosis $(\mathrm{P}=0.001)$, round shoulder $(\mathrm{P}=0.001)$, forward head $(\mathrm{P}=0.002)$ and chest expansion $(\mathrm{P}=0.002)$ between pretest and posttest phases. ShapiroWilk test results indicate normal distribution of data for thoracic kyphosis and round shoulder $(\mathrm{P}>0.05)$, and abnormal distribution of data in forward head and chest expansion in study groups $(\mathrm{P}<0.05)$. Therefore, parametric methods of ANOVA and paired t-test were used to analyze the thoracic kyphosis and round shoulder variables and nonparametric Mann-Whitney U and Wilcoxon tests.

\section{Discussion}

Improvement in angles of forward head, round shoulders and kyphosis and chest expansion size in female computer users with UCS indicated optimal effectiveness of selected corrective exercises with physioball in improving posture.

Table 1. ANOVA results from comparing the mean post-test degrees of round shoulder and thoracic kyphosis between groups

\begin{tabular}{cccccccc}
\hline Variable & Phase & Group & Mean & F & df & Sig. & Eta Squared \\
\hline \multirow{2}{*}{ Thoracic kyphosis } & Pre-test & Control & 49.45 & 63.36 & 1 & $0.001^{*}$ & 0.75 \\
& Post-test & Exercise & 42.94 & & & & 0.12 \\
Round shoulder & Pre-test & Control & 54.34 & & & \\
& Post-test & Exercise & 52.40 & 2.85 & 1 & 0.10 & Journal of \\
& & & & & & Sport Biomechanics
\end{tabular}

Table 2. Mann-Whitney U test results from comparing the mean amounts of chest expansion and forward head before and after exercise

\begin{tabular}{|c|c|c|c|c|}
\hline Variable & Time & $\mathbf{U}$ & Z & Sig. \\
\hline \multirow{2}{*}{ Chest expansion } & Pre-test & 61.50 & -0.83 & 0.55 \\
\hline & Post-test & 12.00 & -3.55 & $0.001^{*}$ \\
\hline \multirow{2}{*}{ Forward head } & Pre-test & 59.00 & -0.80 & 0.47 \\
\hline & Post-test & 28.50 & -2.63 & $0.01^{*}$ \\
\hline
\end{tabular}


Table 3. Paired t-test results from comparing the mean degrees of round shoulder and thoracic kyphosis before and after exercise

\begin{tabular}{cccccccccc}
\hline \multirow{2}{*}{ Variables } & \multicolumn{4}{c}{ Control Group ( $\mathbf{n = 1 2 )}$} & \multicolumn{4}{c|}{ Exercise Group ( $\mathbf{n = 1 2 )}$} \\
\cline { 2 - 9 } & Pre-test & Post-test & T & P & Pre-test & Post-test & T & Sig. \\
\hline thoracic kyphosis & $48.77 \pm 5.88$ & $48.92 \pm 5.66$ & -1.03 & 0.32 & $5.66 \pm 49.99$ & $43-47 \pm 5.08$ & 7.73 & $0.001^{*}$ \\
round shoulder & $54.91 \pm 2.67$ & $53.91 \pm 3.36$ & 0.98 & 0.34 & $56.58 \pm 1.56$ & $52.83 \pm 2.03$ & 7.58 & $0.001^{*}$ \\
\hline & & & & & & \multicolumn{3}{c}{$\begin{array}{c}\text { Journal of } \\
\text { Sport Biomechanics }\end{array}$}
\end{tabular}

Table 4. Wilcoxon test results from comparing the mean amounts of chest expansion and forward head before and after exercise

\begin{tabular}{ccccccccc}
\hline \multirow{2}{*}{ Variable } & \multicolumn{4}{c}{ Control Group $(\mathbf{n}=\mathbf{1 2})$} & \multicolumn{5}{c}{ Exercise Group $(\mathbf{n}=12)$} \\
\cline { 2 - 9 } & Pre-test & Post-test & $\mathbf{Z}$ & Sig. & Pre-test & Post-test & Z & Sig. \\
\hline Chest expansion & $2.75 \pm 0.45$ & $2.79 \pm 0.45$ & -1.73 & 0.08 & $2.91 \pm 0.51$ & $3.73 \pm 0.54$ & -2.95 & $0.003^{*}$ \\
Forward head & $46.83 \pm 0.93$ & $46.58 \pm 0.90$ & -0.37 & 0.70 & $47.08 \pm 0.90$ & $44.91 \pm 1.44$ & -3.10 & $0.002^{*}$ \\
\hline & & & & & & \multicolumn{3}{c}{$\begin{array}{l}\text { Journal of } \\
\text { Sport Biomechanics }\end{array}$}
\end{tabular}

\section{Conclusion}

The present study focused on the muscles involved in this deformity, which was designed and implemented based on Janda's chain reaction theory and the Brugger's gear model. The exercises simultaneously affected all three postural deformities (hyperkyphosis, forward head, round shoulder), and the subjects participated actively and dynamically in the exercises. The use of this training program is recommended for computer users.

\section{Ethical Considerations}

Compliance with ethical guidelines

The present study obtained its ethical approval from the Guilan University of Medical Sciences (Code: IR.GUMS. REC.1397.480) and is a clinical trial registered by the Iranian Registry of Clinical Trials.

Funding

This study was extracted from the master thesis of first author approved by the Department of Sport Injuries and corrective Exercises, University of Guilan, Rasht, Iran.

\section{Authors' contributions}

Conceptualization, methodology, supervision: All authors; Investigation, writing original draft, resources: Parisa Sedaghati. editing \& review: Fariba Javazi and Hasan Daneshmandi.

\section{Conflicts of interest}

The authors declared no conflict of interest.

\section{Acknowledgements}

The authors would like to thank the Deputy for Research of the University of Guilan and all the subjects who sincerely participated in the present study. 


\title{
تأثير تمرينات منتخب اصلاحى بافيزيوبال بر وضعيت ياسجر كاربران رايانه داراى سندرم مثقاطع فوقانى
}

\author{
فريبا جوازى' نه، "بريسا صداقتى' ن، حسن دانشمندى' \\ 1. كروه آسيبشناسى ورزشى وحركات اصلاحي، دانشكده تربيتبنىى وعلوم ورزشى، داثشكاه كيلان، رشت، ايران.
}

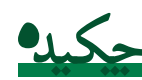

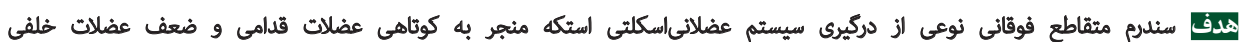

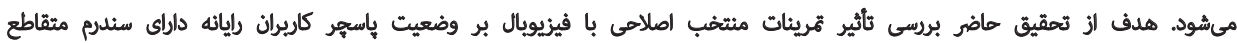$$
\text { فوقانى بود. }
$$

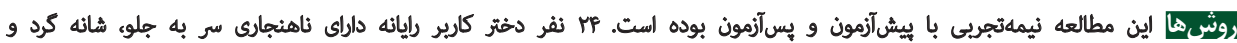

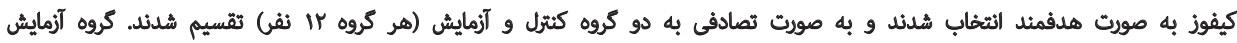

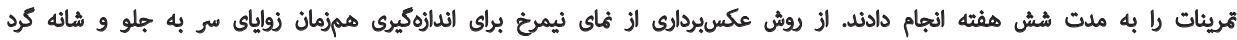

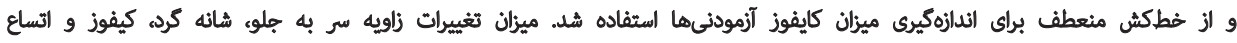

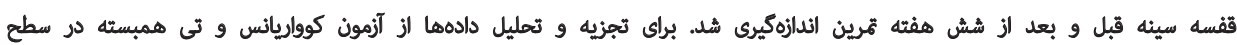

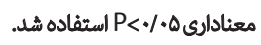

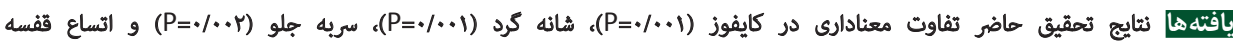

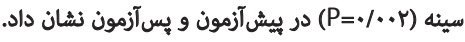

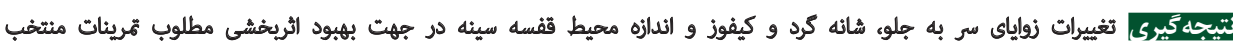

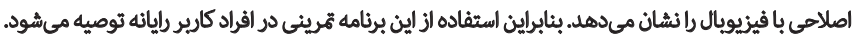

اطلاعات مقاله:

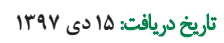

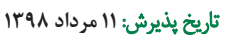

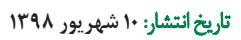

و سبب بروز ناهنجارى شود [F] و اين ثاهنجارى منجر به تغيير

doteo

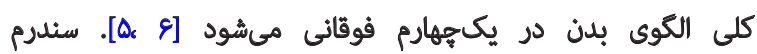

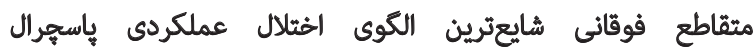

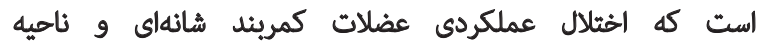

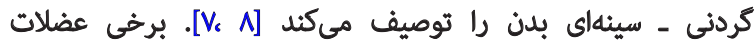

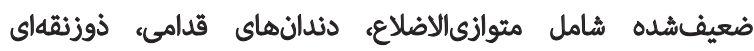

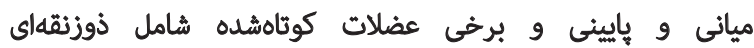

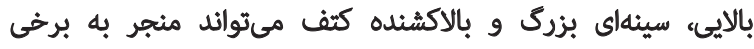

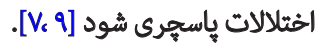

تغييرات وضعيتى خاصى در سندرم متقاطع فوقانى ديده

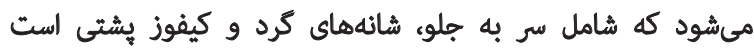

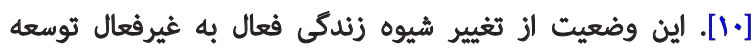

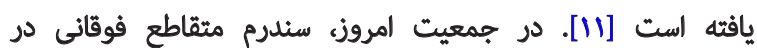

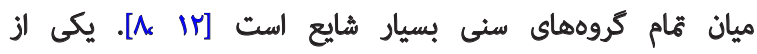

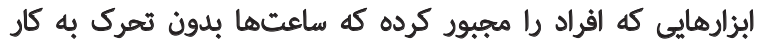

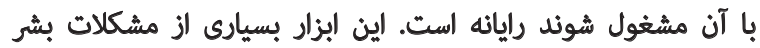

وضعيت بدنى صحيح به نكهدارى طبيعى و موزون بخشهاي

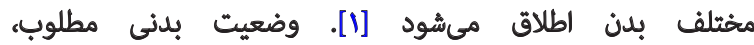

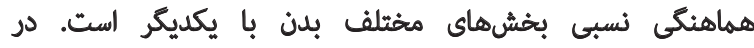

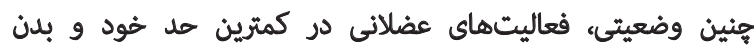

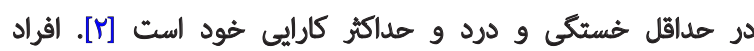

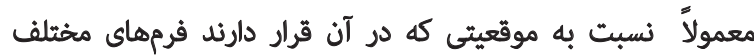

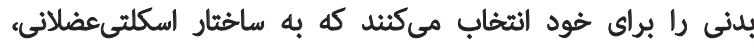

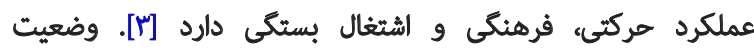

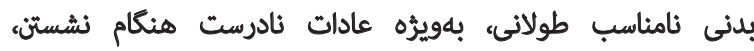

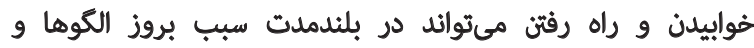

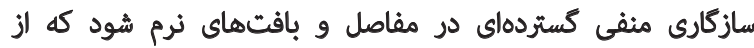

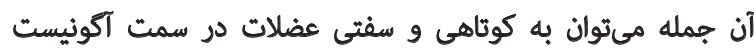

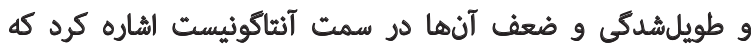

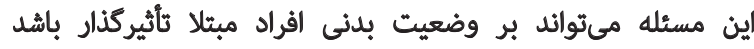




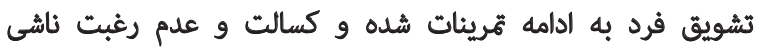

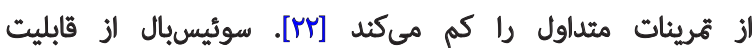

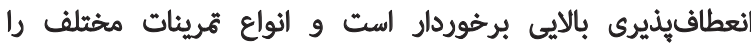

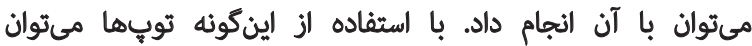

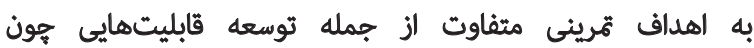

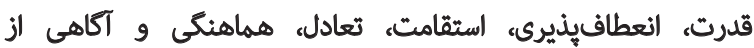

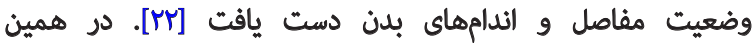

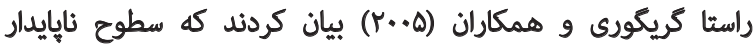

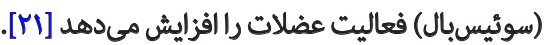

الين كورا و همكاران (1+٪) ثأثير تمرينات نفس كشيدن و

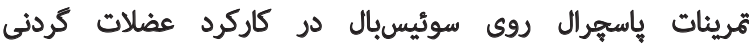

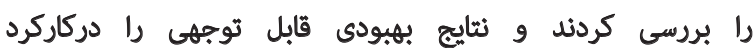

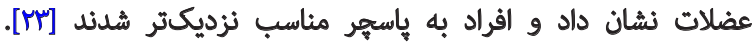

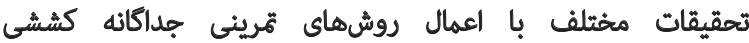

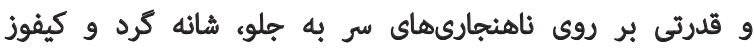

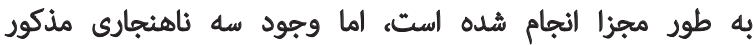

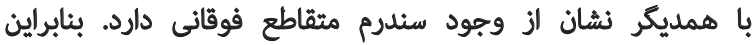

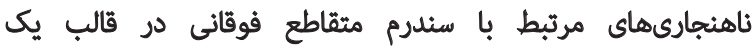

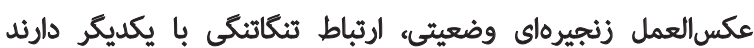

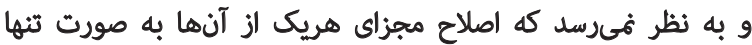

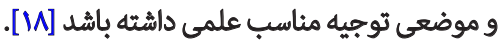
با توجه به اهميتى كه اصلاح وضعيث باسجير دارد با استفاده از حركات اصلاحى مناسب در رفع ناهنجارىهاى وضعيتى و وارئي ارائه

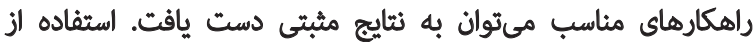

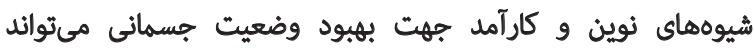

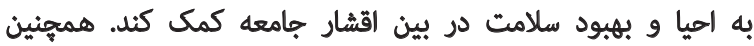
انجام حركات متنوع و جذاب با استفاده از فيزيوبال مئوانداند اشتياق

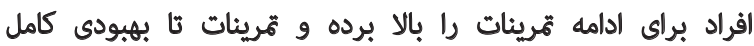

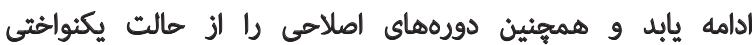

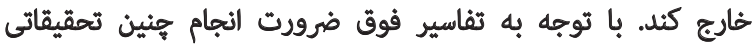

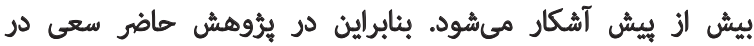

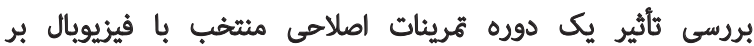
وضعيت ياسيحر زنان داراى سندرم مثقاطع فوقانى شد.

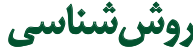

تهقيق حاضر از نوع كارآزمايى بالينى با طرح بيشآزمون و

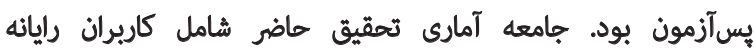

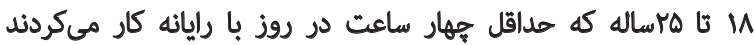

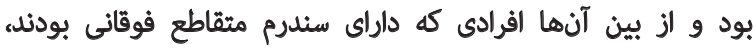

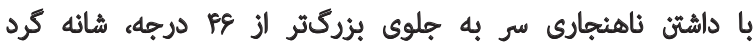

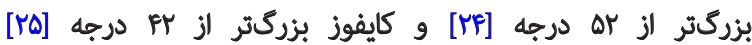

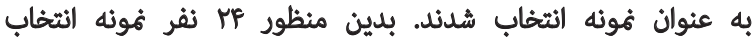

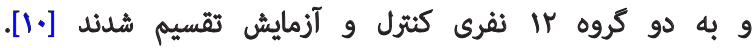

امروزى را حل كرده، ليكن آسيبهايى كه هم|اكنون كريبانكير

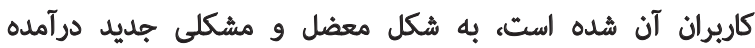

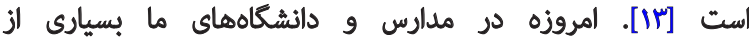

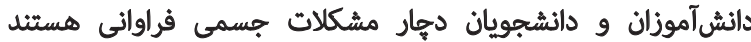

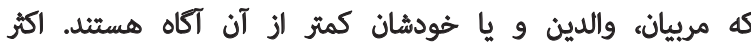

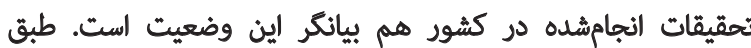

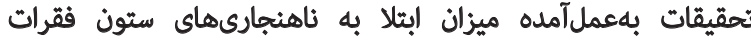

در جامعه دانشجويان نيز شيوع بالايى دارد [IF]

دانشجويان از جمله افرادى هستثد كه روزانه ساعتهاى زيادى را صرف كار با رايانه مىكنند كه در زمان وران كار بار با رايانه

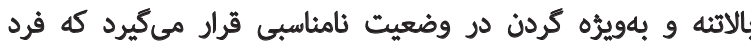

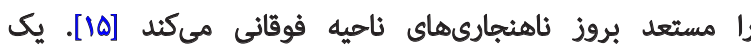

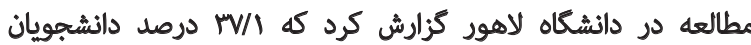

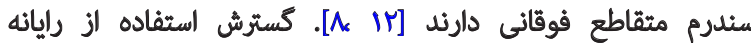

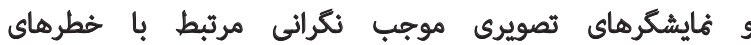

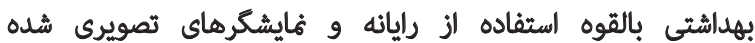

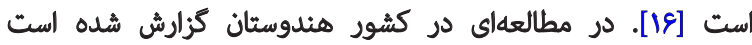

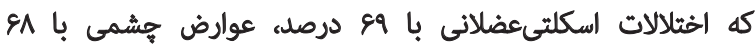

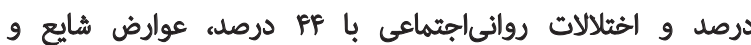

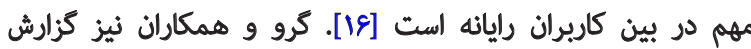

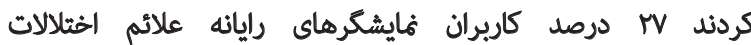

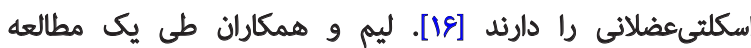

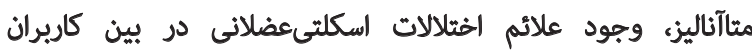

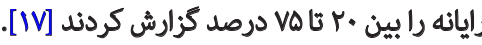

كر ناحيه يشتى، هاييركايفوزيس به معناى افزايش زاويه قوس

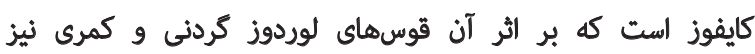

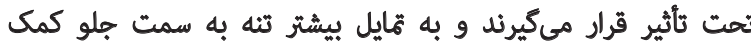

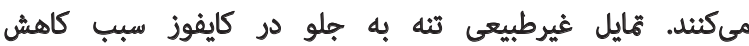

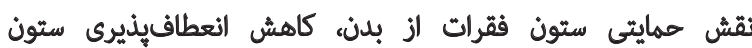

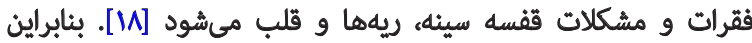

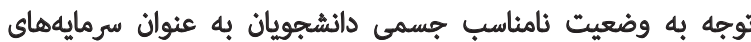

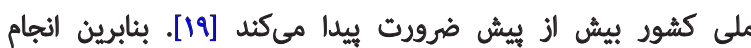

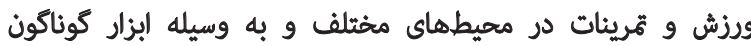

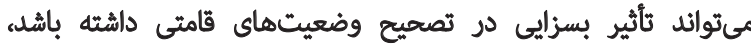

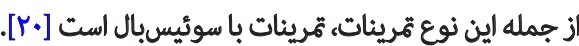

استفاده از اين توبٍ ميتواند باعث فعاليت بيشتر عضلات

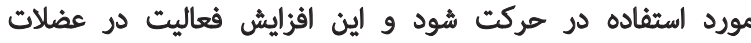

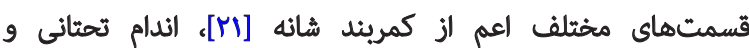

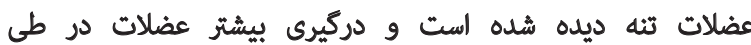

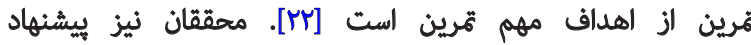

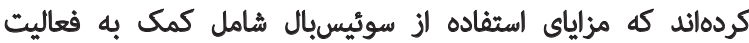

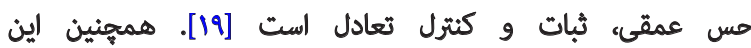

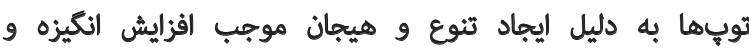


براى اندازمكيرى زاويه كايفوز آزمودثىها الز خطكش منعطف

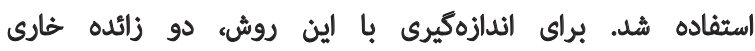

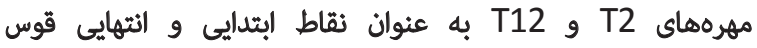

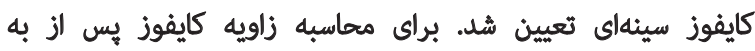

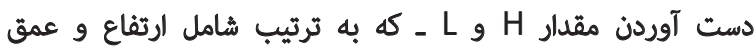

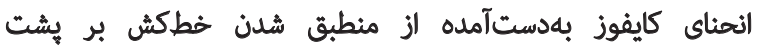

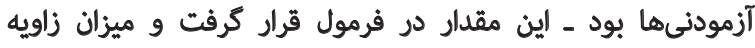

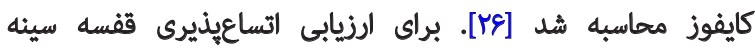

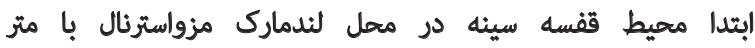

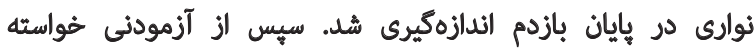

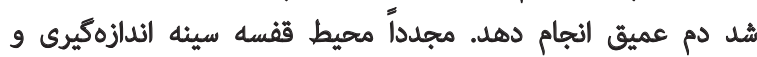
اخخثلاف دو اندازهكيرى ثبت شد [TV]

برنامه تمرينات اصلاحى منتخب با فيزيوبال كه شامل سه مرحله تمرينات كششى، تقويتى و انسجام با بهكاركيرى فيزيوبال

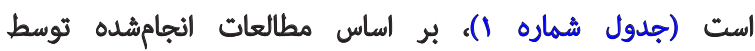

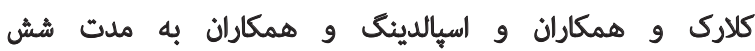

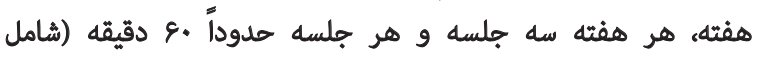

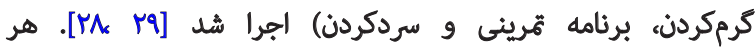

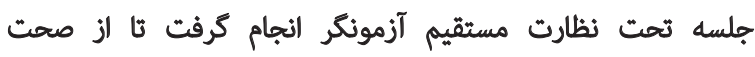

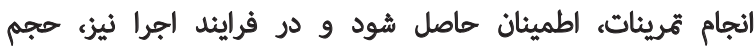

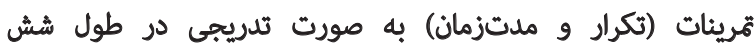

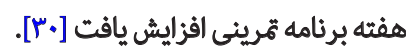

كر اين تحقيق براى تجزيه و تحليل آمارى از نرمافزار SPSS

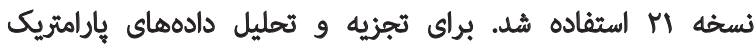

آزمودنىها رضايتنامه كتبى :براى شركت داوطلبانه در تهقيق

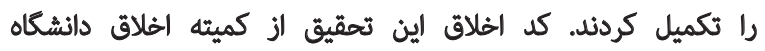

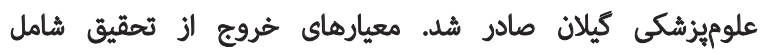

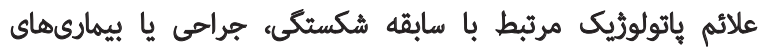

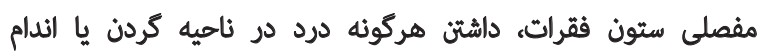

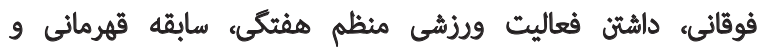
عضويت در تيمهاى ورزشى بود. همجينين علم المام برنامه تمرينى

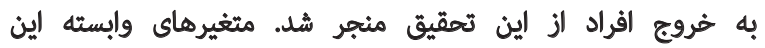
تحقيق شامل سر به جلو، كيفوز، شائه كرد و اتساعيذئيرى قفسيه

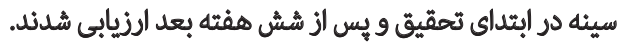

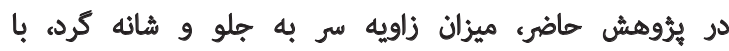

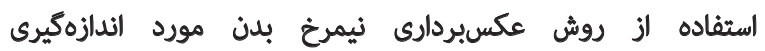

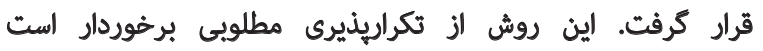

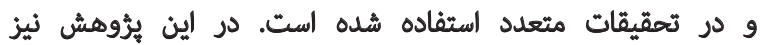

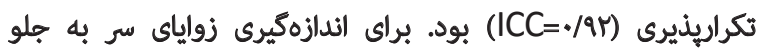

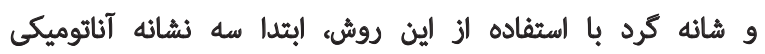
تراكوس كوش و برجستكى أكروميون سمت رائ راست و و همجهنين،

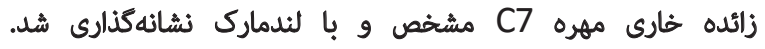

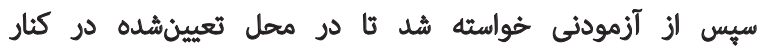

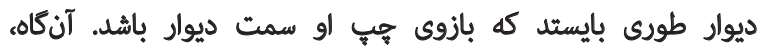

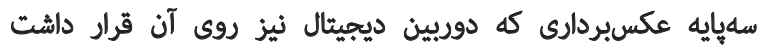

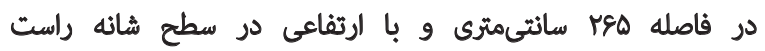

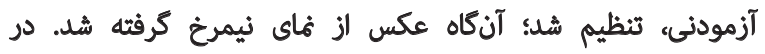

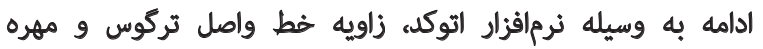

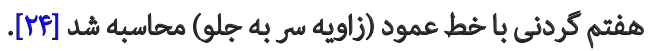

جدول ا. برئمه تمرينات منتخب با فيزيوبال

\begin{tabular}{|c|c|c|c|c|}
\hline هفته ه-9 & هفته F-r & Sفته I-1 & تمرين & \\
\hline "است/|r|ثانيه & آست/r|ثاثيه & آكت/•اثانيه & كثش ستون فقرات & \\
\hline "الست/Y|ثانيه & است/rاثانيه & آنست/•اثانيه & اكستنشن بشت & \\
\hline "آست/Yاثانيه & كاست/rاثانيه & آست/ءاثانيه & كشش يشتى & كششى \\
\hline "است/rاثانيه & كاست/Yاثانيه & זٓست/.اثانيه & كشش جانبى تنه & \\
\hline "الست/rاثانيه & است/rاثانيه & كست/.اثانيه & كشش جانبهاى سينه & \\
\hline 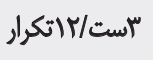 & كاتس/rاتكرار & 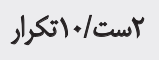 & سويرمن & \\
\hline " الست/Tاتكرار & كاست/rاتكرار & بست/·تكرار & شنا روى توب & \\
\hline " الست/rاتيكرار & كآست/rاتكرار & كابست/.اتكرار & اكستشن كردن & قلمرتى \\
\hline " الست/rاتكرار & 1 آست/rاتكرار & 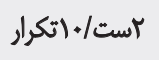 & جين تاى در وضعيت هيار دست و با با توب & \\
\hline " استب/Tاتيكرار & كابت/rاتكرار & | آست/.اتكرار & نكله داشت توب & \\
\hline 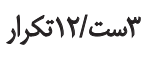 & باست/rاتكرار & | آست/.اتكرار & كومبو روى توب & \\
\hline " است/Tاتيكرال & إست/rاتكرار & 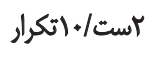 & يرس سينه & 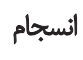 \\
\hline " است/זاتكرار & كاست/rاتكرار & استث/+اتكرار & كومبو روى توب با ريتراكشن كردن & \\
\hline
\end{tabular}

مجله بيومكانيك ورنش 
با توجه به غيرثرمال بودن دادهاي متغيرهاي محيط قفسيه سينه

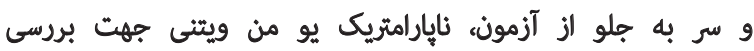

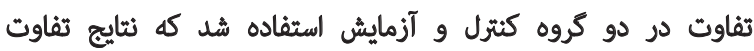

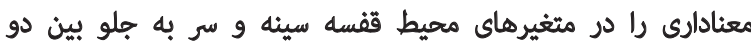

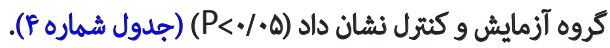
براي بررسى تفاوت در ييشآزمون و بسآزمون در دو كروه

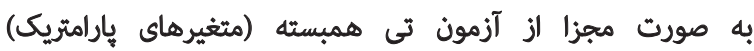

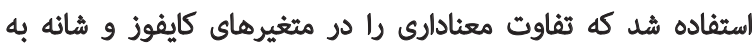

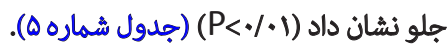

همجينين براى بررسى تفاوت در ييشآزمون و يسآزمون در دو كروه

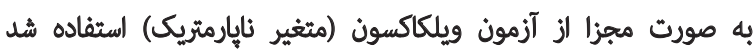

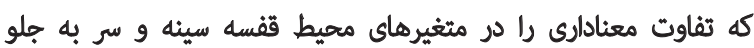

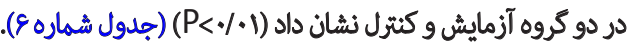

از آزمون كوواريانس و تى همبسته و براي دادههاى ثايارامتريك

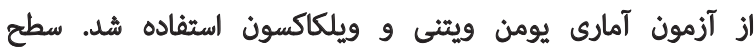
معناداري اين تحقيق هـ/ تعيين شد.

نيانج

آزمون شاييرو ويلك حاكى الز توزيع نرمال در دادههاى كايفوز

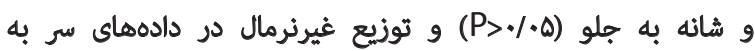

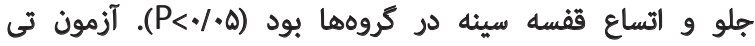

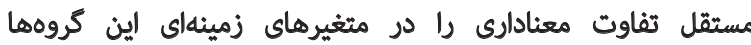
نشان نداد (جدول شماره r). براى مقايسه ميانكين ثنايج بين دو كروه با در نظر كرفتن ييشآزمون

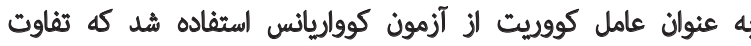

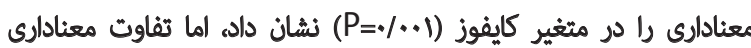

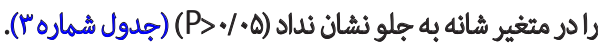

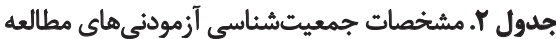

\begin{tabular}{|c|c|c|c|}
\hline \multirow{2}{*}{$\mathbf{P}$} & \multicolumn{2}{|c|}{ مياتكين +انحراف معيار } & \multirow{2}{*}{ متغير } \\
\hline & كروه كتترل & توره آزمايش & \\
\hline.$/ \Delta \Delta$ & $\mathrm{r} / \Delta \mathrm{\Delta} \pm \mathrm{V} / \mathrm{M}$ & $M / * \pm V / \Delta Y$ & سن (سال) \\
\hline $.18 \Delta$ & V/grt+1.P & $1 / \& \Psi \pm=1 . r$ & قد (سائتىمتر) \\
\hline . Iat & $\Delta V / V \Delta \pm Y / Q \Delta$ & $\Delta E / A T \pm Y M T$ & وزن (كيلوكرم) \\
\hline.$|\Delta|$ & $r / q . \pm r / 1 q$ & $r / M f \pm 1 / q r$ & شاخص توده بلدنى \\
\hline
\end{tabular}

مجله بيومكانيك ولنش

جدول ऍ. نتايج تحليل كوواريائس جهت مقايسه متغيرها در يسآزمون بين كروهها

\begin{tabular}{|c|c|c|c|c|c|c|c|}
\hline Eta & $\mathbf{P}$ & درجه آزادى & $\mathbf{F}$ & ميانكين" & توروه & مرحله آزمون & متغير \\
\hline \multirow[b]{2}{*}{ - Na } & \multirow[b]{2}{*}{$.1 .01^{*}$} & \multirow[b]{2}{*}{1} & \multirow[b]{2}{*}{ E } & $r q / 40$ & كثترل & يس آزمون & \multirow[b]{2}{*}{ كايفوز } \\
\hline & & & & $r r / q u$ & أزمايش & يس آَّون & \\
\hline \multirow{2}{*}{.$/ N$} & \multirow{2}{*}{.11} & \multirow{2}{*}{1} & \multirow{2}{*}{ r/NQ } & $\Delta F / M F$ & كتترل & يس آزمون & \multirow{2}{*}{ شائه به جلو } \\
\hline & & & & $\Delta r / F$. & آزمايش & يس آزمون & \\
\hline
\end{tabular}

جدول F. نتايج آزمون يو من ويتنى جهت مقايسه متغيرهاى محيط قفسه سينه و سر به جلو در يُيش آزمون و يّآزمون بين كروهها

\begin{tabular}{|c|c|c|c|c|}
\hline $\mathbf{P}$ & $\mathbf{z}$ & $\mathbf{U}$ & زمان & مثغير \\
\hline$\cdot / \Delta \Delta$ & $-+/ A r$ & SI/A. & تيش أزمون & \multirow[b]{2}{*}{ مجيط قفسله سينه } \\
\hline $.1 .+1^{*}$ & $-r / \Delta \Delta$ & $\| r / .$. & بسآزمون & \\
\hline . pr & $-\cdot / A$ & $\Delta V_{*}$. & يبش أزمون & \multirow{2}{*}{ سر به جلو } \\
\hline $.1 \cdot 1^{*}$ & - T/ET & TND. & يسآزمون & \\
\hline
\end{tabular}

مجله بيومكانيك ورنث 
جدول ه. تفاوت ميائكين متغيرهاى كايفوز و شائه به جلو در آزمودئىها قبل و بعد از اعمال يروتكل تمرينى

\begin{tabular}{|c|c|c|c|c|c|c|c|c|}
\hline \multicolumn{4}{|c|}{ كروه أزمايش (rا نفر) } & \multicolumn{4}{|c|}{ كروه كتترل (rا نفر) } & \multirow{3}{*}{ تئروه } \\
\hline \multicolumn{4}{|c|}{ ميائكين +انحراف معيار } & \multicolumn{4}{|c|}{ ميانكين \|نحراف معيار } & \\
\hline $\mathbf{P}$ & $\mathbf{T}$ & بِس آزمون & ييش آزمون & $\mathbf{P}$ & T & يس آزمون & يميش آزمون & \\
\hline$\%$ & $V / M$ & $\varphi r / R V \pm \Delta / \cdot \Lambda$ & pq/११ะه/\&8 &.$/ \pi$ & $-1 / \cdot r$ & PNAY $\pm \Delta / 98$ & $P M W \pm \Delta / M$ & كايفوز \\
\hline$\% 1^{*}$ & $V / \Delta A$ & $\Delta Y / A T \pm Y / \cdot r$ & $\Delta F / \Delta A \pm 1 / \Delta F$ &.$/ M \varphi$ &.$/ 44$ & $\Delta \Gamma / Q \mid \pm \Gamma / T \varepsilon$ & $\Delta F / Q 1 \pm r / q V$ & شانه به جلو \\
\hline
\end{tabular}

مجله بيومكانيك وزنش

جدول و تفاوت ميانكين متغير تعادل عملكردى در آزمودنىها قبل و بعد از اعمال بروتكل تمرينى

\begin{tabular}{|c|c|c|c|c|c|c|c|c|}
\hline \multicolumn{4}{|c|}{ كروه آزمايش (T/ نفر) } & \multicolumn{4}{|c|}{ كروه كتترل (rا نفر) } & \multirow[b]{3}{*}{ كروه } \\
\hline \multicolumn{4}{|c|}{ ميانكين \$انحراف معيار } & \multicolumn{4}{|c|}{ ميانكين土|نحراف معيار } & \\
\hline $\mathbf{P}$ & $\mathbf{z}$ & يس آزمون & ييش آزمون & $\mathbf{P}$ & $z$ & بس آزمون & ييش آزمون & \\
\hline$\%$ & $-r / 9 \Delta$ & $r / M \Psi \pm \cdot / \Delta F$ & $r / 9| \pm . / \Delta|$ & $\% 1 \cdot 1$ & $-1 / N$ & $r / V q \pm \cdot / F \Delta$ & $r / V \Delta \pm \cdot / \uparrow \Delta$ & محيط ققسه سينه \\
\hline$\%$ & $-r / 1$. & $P \varphi / 91 \pm 1 / 4$ & $\mid \mathrm{V} / \cdot \wedge \pm . / 9$. &.$/ N$ &.$- / r V$ & PQ/ $/ \Delta A \pm . / 9$. & 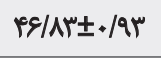 & سر به جلو \\
\hline
\end{tabular}

مجله بيومكانيك وزنش

سر به جلو يكى از رايجترين انحرافات وضعيتى در ربع فوقانى

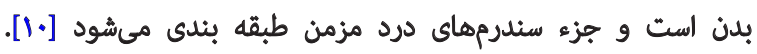

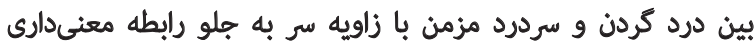

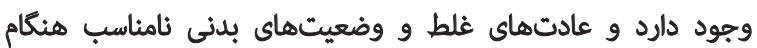

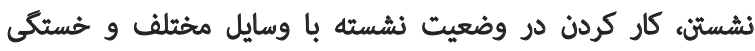

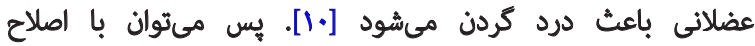

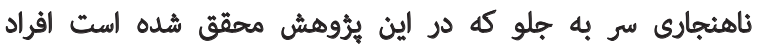

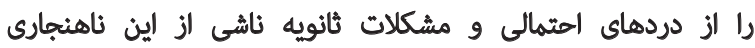

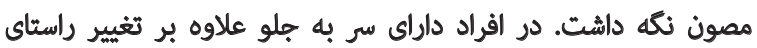

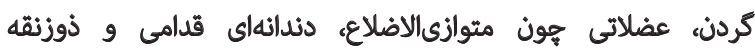

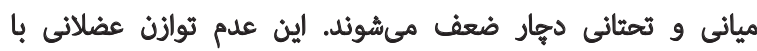

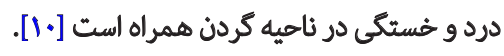

در همين راستا در يُوهشى كه نيكروان و و همكاران (IITP) السكلثيعضلاني و درد ناحيه كردن و كمربند شانه كاربران رايائه

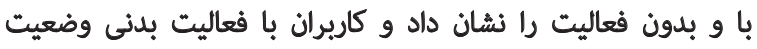

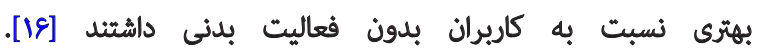

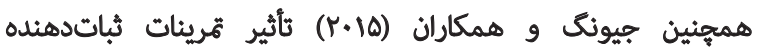

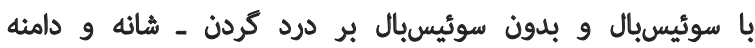

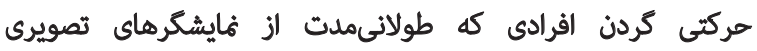

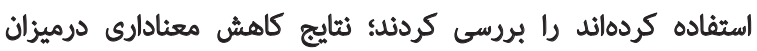

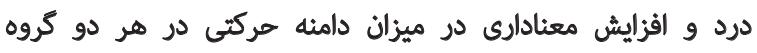

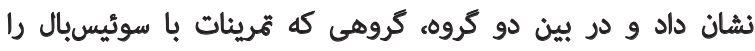

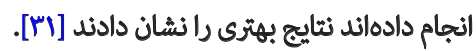

تحقيقات ثشان مىدهد تركيب ورزشهاي ثباتى و متمرين

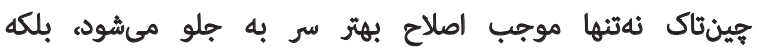

نتايج اين مطالعه نشان داد تحت ثأثير شش هفته ثمرينات

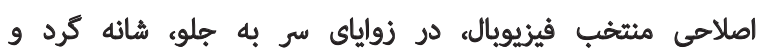

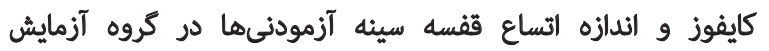

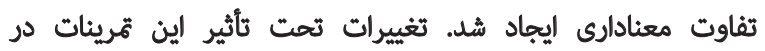

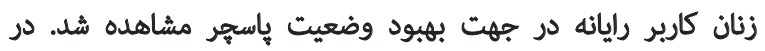

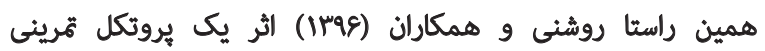
مبتنى بر اصول NASM بر سندرم متقاطع فوقانى آسيبديدكان رئن

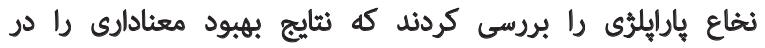

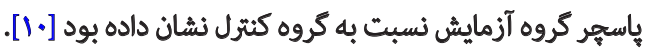
رجالاكسيمى و همكاران (1/. (T) اثريخشى دو روش ثمرينى

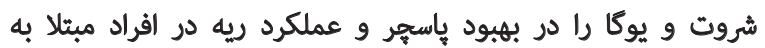

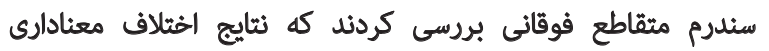

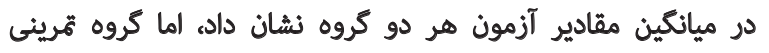

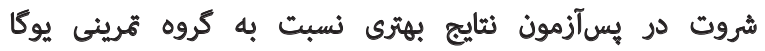

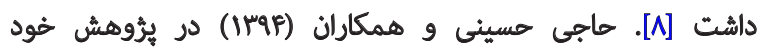

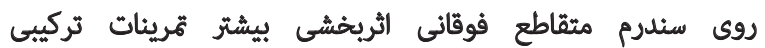
نسبت به تمرينات مجزاي كششى و قدرتى را نشان دادند [T]].

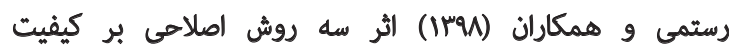

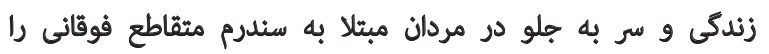

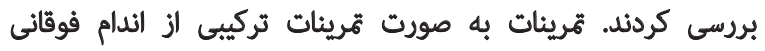

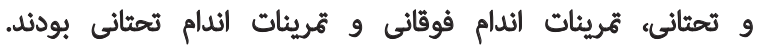

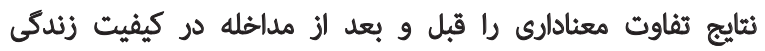

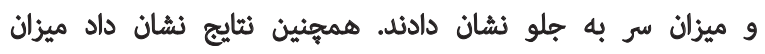

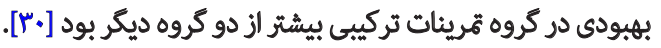


زنجيره وضعيتى در مهرهها رغ مغدهد. الز آنجا كه نواحي

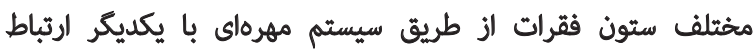

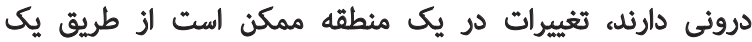

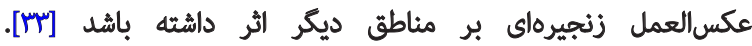

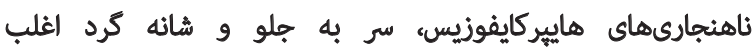

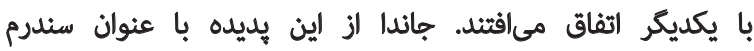

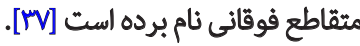

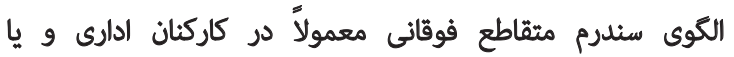

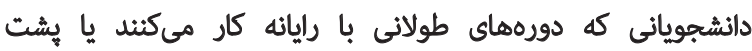

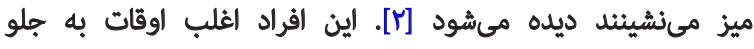

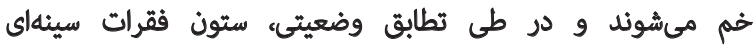

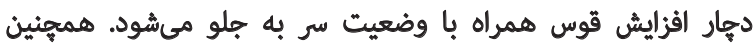

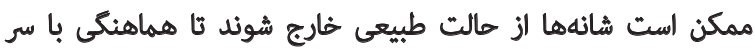

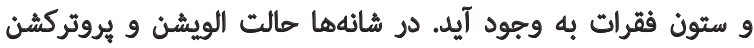

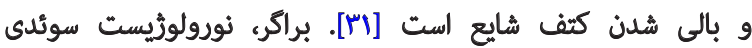

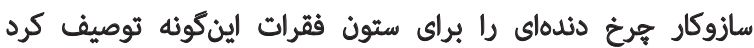

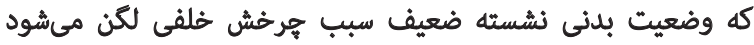

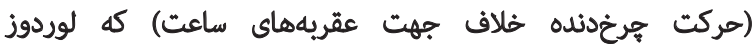

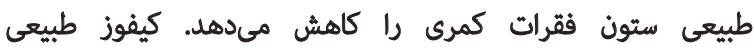

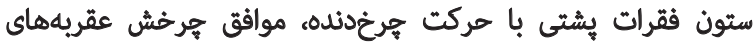

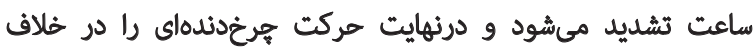

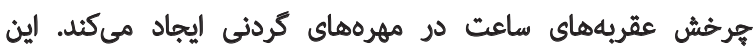

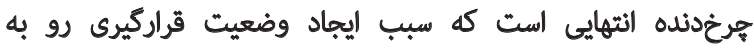

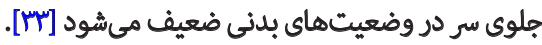

تمرينات بروهش حاضر بر عضلات دركير در اين ناهنجارى منمركز

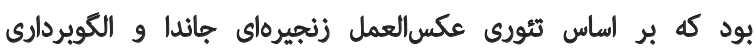

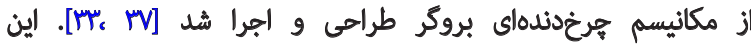

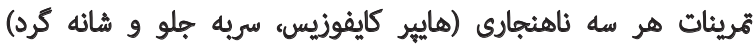

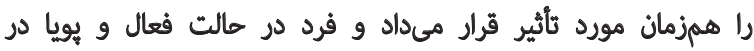

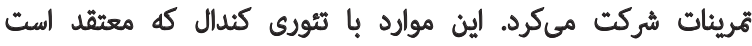

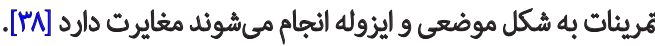
حاجى حسينى و همكاران (99"1)، در مطالعه خود روى سندرم

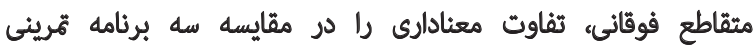
هجزاي قدرتي و كششى و يك برنامه جامع، روى شانه كرد نيافتند

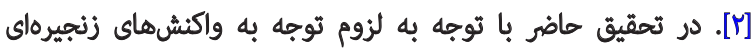

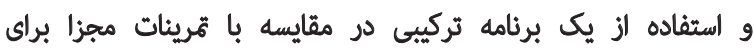

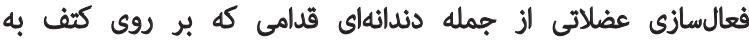

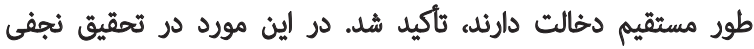

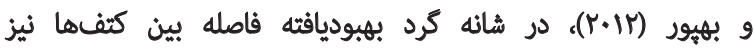

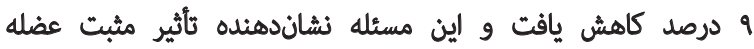

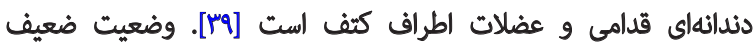

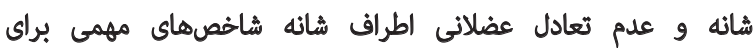

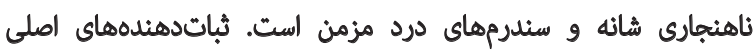

موجب هايدالوى مؤثرتر و ثبات ياسجر مىشود [.1]. روشنى

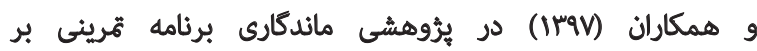

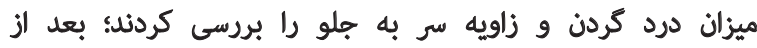

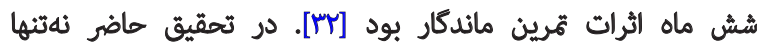

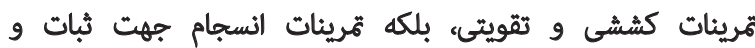

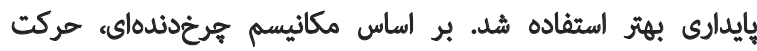

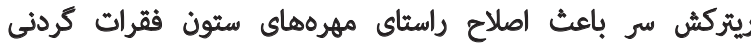

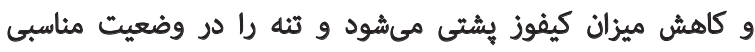

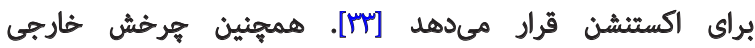

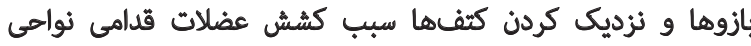

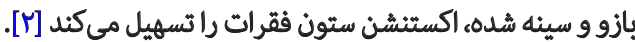
يكى از تمرينات انسجامى كه در يُوهش حاضر كنجانده شد

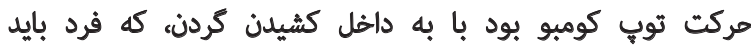

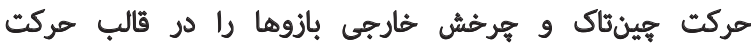

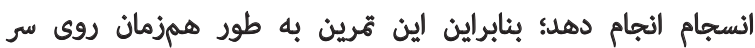

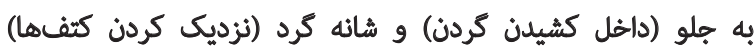

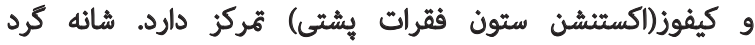

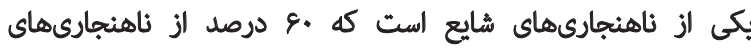

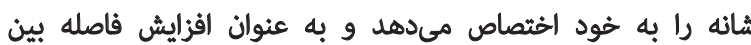

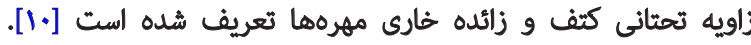

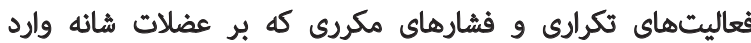

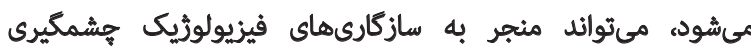

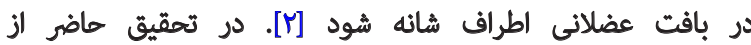

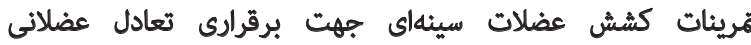

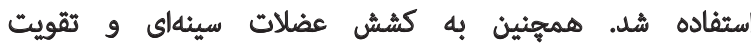

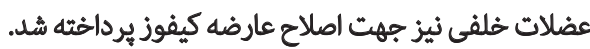

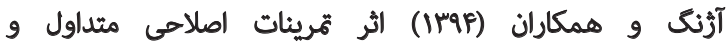

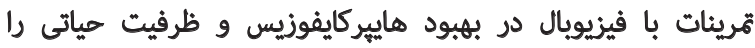

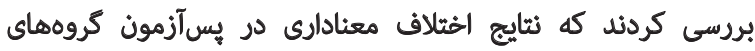

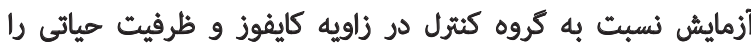

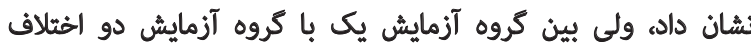

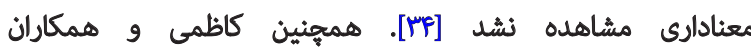

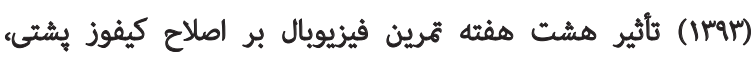

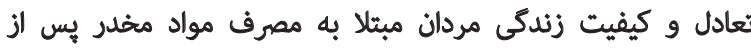

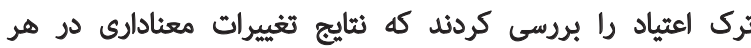

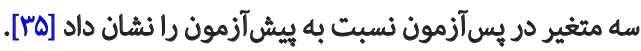
با توجه به تأثير كيفوز روى اتساع قفسه سينه، اين محدوديت

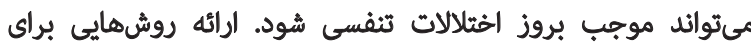

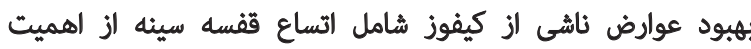

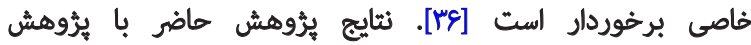

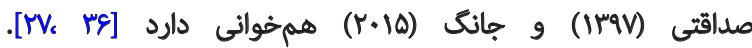

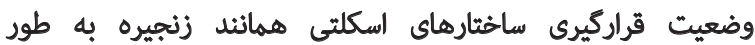

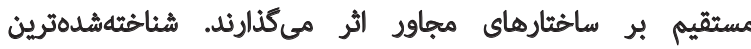


جوازي، كروه آهيب شناسى ورزشى و حركات اصلاحى، دانشكده تربيتبدنى و علوم ورزشى، دانشعاه كيلان است.

$$
\text { مشاركتنويسندكَان }
$$

مفهومسازى، روششئاسي و نظارت: تمامى نويسندكان. برربيى

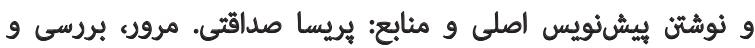
ويرايش: فريبا جوازى و حسن دانشمندى. ئنسئ.

$$
\text { تعارض منافع }
$$

بنابر اظهار نويسندكان اين مقاله تعارض منافع ندارد.

$$
\text { تشكر وقدردانى }
$$

بلدينوسيله از معاونت بثزوهشى دانشكاه كيلان و وقمامى

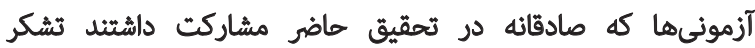
و قدردانى مي شوده.
كتف متوازي|الاضلاع، بالإبرنده كتف، ذوزنقه و دندانهاى قدافى هستند.

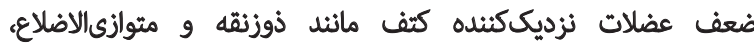

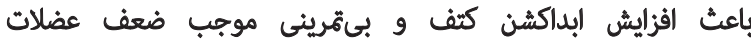

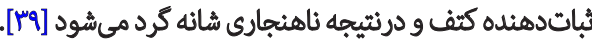

از آنجا كه هرخش در مهرههاي كردنى ميثوائد آسانتر از رهاز

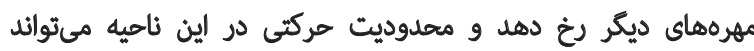

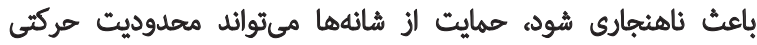

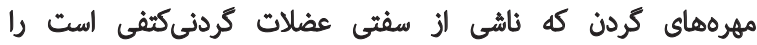

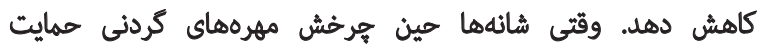

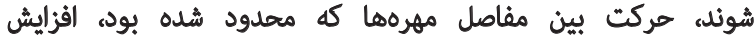

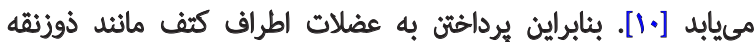

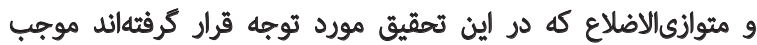

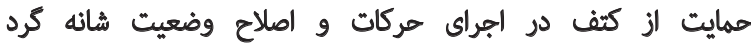

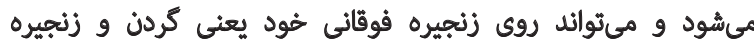
تحثائى خود يعثى ستون فقرات يشتى تاثير مثبت اعمال كند.

به طور كلى اين كونه مىتوان عنوان كرد كه طراحى و الجراى

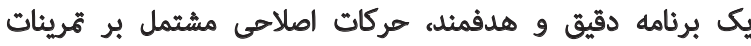

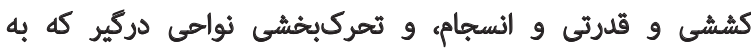

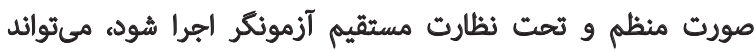
اثربخشى مطلوبى در اصلاح ناهنجارى اقراد مبتلا داشته باشد.

\section{نتيجلكيرى نهايى}

براساس نتايج هيزوهش حاضر، ميتوان نتيجه كرفت كه ثمرينات

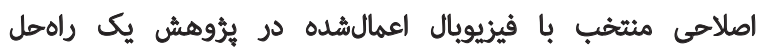

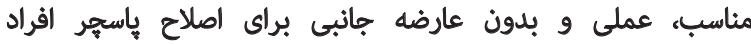

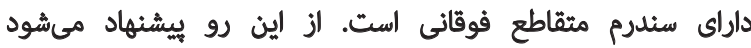

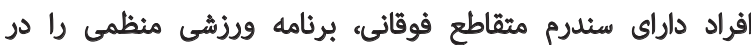

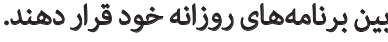

از جمله محدوديتهاي اين مطالعه ميتوان به محدود بودن

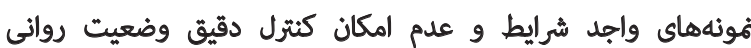
و انكيزشى افراد در فرايند تحقيق الشاره كرد.

ملاحظات اخلاقى

$$
\text { ييروى از اصول اخلاق ثيروهش }
$$

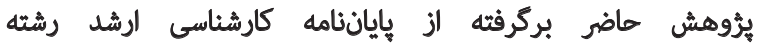

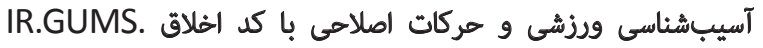

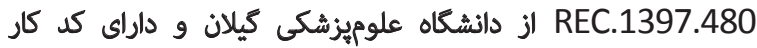
آزمايي بالينى در سامانه كارآزمايى بالينى ايران الست.

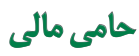

اين مقاله بركرقته از يايانه كارشناسىارشد نويسنده اول فريبا 


\section{References}

[1] Houglum PA. Therapeutic exercise for athletic injuries. Champaign: Human Kinetics; 2001. https://books.google.com/ books?id=z358QgAACAAJ\&dq

[2] Hajihosseini E, Norasteh AA, Shamsi A, Daneshmandi H. [The comparison of effect of three programs of strengthening stretching and comprehensive on upper crossed syndrome (Persian)]. Research in Rehabilitation Sciences. 2015; 11(1):51-61. http://jrrs.mui.ac.ir/index.php/jrrs/ article/view/2149

[3] Johnson G, Bogduk N, Nowitzke A, House D. Anatomy and actions of the trapezius muscle. Clinical Biomechanics. 1994; 9(1):44-50. [DOI:10.1016/0268-0033(94)90057-4]

[4] Nadler SF, Malanga GA, Bartoli LA, Feinberg JH, Prybicien M, Deprince M. Hip muscle imbalance and low back pain in athletes: Influence of core strengthening. Medicine \& Science in Sports \& Exercise. 2002; 34(1):9-16. [DOI:10.1097/00005768-200201000-00003] [PMID]

[5] Penha PJ, Baldini M, João SMA. Spinal postural alignment variance according to sex and age in 7-and 8-year-old children. Journal of Manipulative and Physiological Therapeutics. 2009; 32(2):154-9. [DOI:10.1016/j. jmpt.2008.12.009] [PMID]

[6] Daneshmandi H, Harati J, Fahim Poor S. Bodybuilding links to upper crossed syndrome. Physical Activity Review. 2017; 5:124-31. [DOI:10.16926/par.2017.05.17]

[7] Moore MK. Upper crossed syndrome and its relationship to cervicogenic headache. Journal of Manipulative and Physiological Therapeutics. 2004; 27(6):414-20. [DOI:10.1016/j.jmpt.2004.05.007] [PMID]

[8] Rajalaxmi V, Paul J, Nithya M, Chandra LS, Likitha B. Effectiveness of three dimensional approach of schroth method and yoga on pulmonary function test and posture in upper crossed syndrome with neck Pain-A double blinded study. Research Journal of Pharmacy and Technology. 2018; 11(5):1835-9. [DOI:10.5958/0974-360X.2018.00341.4]

[9] Morris CE, Bonnefin D, Darville C. The torsional upper crossed syndrome: A multi-planar update to Janda's model, with a case series introduction of the mid-pectoral fascial lesion as an associated etiological factor. Journal of Bodywork and Movement Therapies. 2015; 19(4):6819. [DOI:10.1016/j.jbmt.2015.08.008] [PMID]

[10] Roshani S, Mahdavinejad R, Ghanizadehesar N. [The effect of a NASMbased training protocol on upper cross syndrome in paraplegia spinalcord injury patients (Persian)]. Journal of Ilam University of Medical Sciences. 2018; 25(6):73-85. [DOI:10.29252/sjimu.25.6.73]

[11] Łukasik E, Targosiński P, Szymański M, Letkiewicz-Ryłów O, Styczeń P, Wychowański M. [Comparing the effectiveness of myofascial techniques with massage in persons with upper crossed syndrome (preliminary report) (Polish-English)]. Postępy Rehabilitacji. 2017; (2):53-67. [DOI:10.1515/rehab-2015-0067]

[12] Mubeen I, Malik S, Akhtar W, lqbal M, Asif M, Arshad A, et al. Prevalence of upper cross syndrome among the medical students of University of Lahore. International Journal of Physiotherapy. 2016; 3(3):381-4 [DOI:10.15621/ijphy/2016/v3i3/100851]

[13] Hoseinpour S. [Relation between lumbar lordosis and thoracic spine with back pain and neck pain in high school girls in Tehran (Persian)] [MSc. thesis]. Tehran: Payame Noor University South Tehran; 2017.

[14] Adedoyin RA, Idowu BO, Adagunodo RE, Owoyomi AA, Idowu PA. Musculoskeletal pain associated with the use of computer systems in Nigeria. Technology and Health Care. 2005; 13(2):125-30. [DOI:10.3233/ THC-2005-13206] [PMID]
[15] Tafakhor Z. [Investigating the prevalence of upper crossed syndrome in student users of computer and its relation with their neck pain (Persian) [MSc. thesis]. Tehran: Allameh Tabataba'i University; 2014. http://d-lib. atu.ac.ir/site/catalogue/77676

[16] Nikravan Golsefidi F, Ebrahimi Atri A, Hashemi Javaheri AA. [A comparison of musculoskeletal disorders of neck and shoulder girdle in male computer users with and without physical activity (Persian)]. Journal of Sport Medicine. 2015; 7(2):205-20. [DOI:10.22059/JSMED.2015.56541]

[17] Naqvi M, Yasmeen S. Association of prolong sitting with common musculoskeletal disorders: A study determining prevalence of work related musculoskeletal disorders due to poor posture and bad ergonomics. Rīga, LV: LAP LAMBERT Academic Publishing; 2017. https://www. amazon.com/Association-Prolong-Sitting-Musculoskeletal-Disorders/ dp/6202003251

[18] Ghaem H, Borhani Haghighi A, Zeighami B, Dehghan A. [Validity and reliability of the Persian version of the Parkinson Disease Quality of Life (PDQL) questionnaire (Persian)]. Journal of Kerman University of Medical Sciences. 2010; 16(1):49-58. http://jkmu.kmu.ac.ir/article_17247. $\mathrm{html}$

[19] Illi U. Balls instead of chairs in the classroom? Swiss Journal of Physical Education. 1994; 6:37-9. https://ci.nii.ac.jp/naid/20000909888/

[20] Hoseini SM. [Comparison of water and physioball trainings on balance in the blind male students (Persian)] [MSc. thesis]. Mashhad: Ferdowsi University of Mashhad; 2015. http://library.um.ac.ir/index. php?option=com_lib\&view=docinfo\&type=2\&DoclD=59956\&lang=fa

[21] Lehman GJ, MacMillan B, MacIntyre I, Chivers M, Fluter M. Shoulder muscle EMG activity during push up variations on and off a Swiss ball. Dynamic Medicine. 2006; 5:7. [DOI:10.1186/1476-5918-5-7] [PMID] [PMCID]

[22] Sahami M. [The effect of sit-up exercises on different surfaces (swissball and conventional floor) on abdominal muscle strength, balance and running economy in non-athlete women (Persian)] [MSc. thesis]. Tehran: Shahid Beheshti University. 2009. http://library.sbu.ac.ir/islandora/ object/thesis\%3A26465

[23] Corrêa ECR, Bérzin F. Mouth breathing syndrome: Cervical muscles recruitment during nasal inspiration before and after respiratory and postural exercises on Swiss Ball. International Journal of Pediatric Otorhinolaryngology. 2008; 72(9):1335-43. [DOI:10.1016/j.jijporl.2008.05.012] [PMID]

[24] Thigpen CA, Padua DA, Michener LA, Guskiewicz K, Giuliani C, Keener $J D$, et al. Head and shoulder posture affect scapular mechanics and muscle activity in overhead tasks. Journal of Electromyography and Kinesiology. 2010; 20(4):701-9. [DOI:10.1016/j.jelekin.2009.12.003] [PMID]

[25] Morningstar MW. Cervical hyperlordosis, forward head posture, and lumbar kyphosis correction: A novel treatment for mid-thoracic pain Journal of Chiropractic Medicine. 2003; 2(3):111-5. [DOI:10.1016/ S0899-3467(07)60055-X]

[26] Rajabi R, Seidi F, Mohamadi F. Which method is accurate when using the flexible ruler to measure the lumbar curvature angle? Deep point or mid point of arch. World Applied Sciences Journal. 2008; 4(6):849-52. https://www.researchgate.net/publication/242320422

[27] Jung JH, Moon DC. The effect of thoracic region self-mobilization on chest expansion and pulmonary function. Journal of Physical Therapy Science. 2015; 27(9):2779-81. [DOI:10.1589/jpts.27.2779] [PMID] [PMCID]

[28] Spalding A, Kelly LE. Fitness on the ball: A core program for brain and body. Champaign, IL: Human Kinetics; 2010. https://books.google.com/ books?id=ede92AEjBIYC\&dq 
[29] Clark M, Lucett S. NASM essentials of corrective exercise training. Philadelphia, PA: Lippincott Williams \& Wilkins; 2010. https://books.google. com/books?id=tZGIM2xzeSwC\&dq

[30] Rostamizalani F, Ahanjan Sh, Rowshani S, Bagherian Dehkordi S, Fallah A. [Comparison of the effects of three corrective exercise methods on the quality of life and forward head of men with upper cross syndrome (Persian)]. Journal of Paramedical Sciences \& Rehabilitation. 2019; 8(1):26-36. [DOI:10.22038/JPSR.2019.27480.1717]

[31] Ahn JA, Kim JH, Bendik AL, Shin JY. Effects of stabilization exercises with a Swiss ball on neck-shoulder pain and mobility of adults with prolonged exposure to VDTs. Journal of Physical Therapy Science. 2015; 27(4):9814. [DOI:10.1589/jpts.27.981] [PMID] [PMCID]

[32] Roshani S, Rostamizalani F, Ghanizade N, Mohammad Ali Nasab Firozjah $E$, Sokhtezari $Z$. [Study of the persistence effect of two exercises controlling the scapula and corrective movements on neck pain and angle of head in males with forward head (Persian)]. Journal of llam University of Medical Sciences. 2019; 27(1):148-60. [DOI:10.29252/ sjimu.27.1.148]

[33] Page P, Frank CC, Lardner R. Assessment and treatment of muscle imbalance: The Janda approach. Champaign, IL: Human Kinetics; 2009. https://www.amazon.com/Assessment-Treatment-Muscle-ImbalanceApproach/dp/0736074007

[34] Azhang M, Khayyam Bashi Kh, Fazel AA, Bagheri L, Emamdoost S, Otadi Kh. [Comparison effect of conventional corrective exercise and physioball exercise on improvement of hyperkyphosis and vital capacity in female students (Persian)]. Journal of Modern Rehabilitation. 2016; 9(S2):168-76. http://mrj.tums.ac.ir/article-1-5436-en.html

[35] Kazemi AA, Mahdavinejad R, Ghasemi GA, Sadeghi M. [Effects of an 8-week exercise with physioball on the correction of thoracic kyphosis, balance and quality of life in addicted men after quitting drugs (Persian)]. Journal of Research in Rehabilitation Sciences. 2013; 9(2):328-37. https://www.sid.ir/fa/journal/ViewPaper.aspx?ID=196745

[36] Sedaghati P, Saki F, Mohamadi B. [Investigating the effect of six weeks of functional kinesio taping on body posture and spinal function of elderly women (Persian)]. Complementary Medicine Journal. 2019; 8(4):3457-66. http://cmja.arakmu.ac.ir/article-1-605-en.html

[37] Seidi F, Rajabi R, Ebrahimi E, Alizadeh MH, Daneshmandi H. [The ef fect of a 10-week selected corrective exercise program on postural thoracic kyphosis deformity (Persian)]. Sport Medicine. 2013; 5(10):5-22. https://www.magiran.com/paper/1159883?lang=en

[38] Shiravi S, Letafatkar A, Bertozzi L, Pillastrini P, Khaleghi Tazji M. Efficacy of abdominal control feedback and scapula stabilization exercises in participants with forward head, round shoulder postures and neck movement impairment. Sports Health. 2019; 11(3):272-9. [DOI:10.1177/1941738119835223] [PMID]

[39] Najafi M, Behpoor N. [The effects of a selective corrective program on the scapula and shoulder joint posture in girls with rounded shoulder (Persian)]. Journal of Sport Medicine. 2013; 4(2):31-47. [DOI:10.22059/ JSMED.2013.30058 